\title{
El Campo de la Práctica en Geografía. Una formación que trasciende el aula universitaria $^{1}$
}

\author{
María Graciela Di Franco² y Stella Maris Leduc ${ }^{3}$ \\ Universidad Nacional de La Pampa \\ @ [ chdifranco@gmail.com.ar ] [ leduc.stellamaris@gmail.com ]
}

DOI: http://dx.doi.org/10.19137/huellas-2017-2115

\section{Introducción}

n el año 2009, la Facultad de Ciencias Humanas de la Universidad - Nacional de La Pampa modificó los planes de estudio de todos los Profesorados que allí se dictan. Se aprueba un plan con treinta asignaturas, distribuidas en cinco años (excepto Profesorado en Inglés que lo organiza en cuatro) y tres asignaturas por cuatrimestre. Se prescribe también una formación en cuatro campos, tres de los cuales estaban definidos en los planes anteriores: Formación General, Formación Específica y Formación

1 En este trabajo se recupera la presentación de las autoras en la Mesa del Campo de las Prácticas desarrollada en las V Jornadas de Geografia, Docencia e Investigación, organizadas por el Instituto y el Departamento de Geografía de la Universidad Nacional de La Pampa, en octubre de 2016.

2 Magíster en Evaluación (UNLPam). Especialista en Estudios Sociales y Culturales (UNLPam). Docente de Práctica Curricular y Didáctica de la Facultad de Ciencias Humanas de la UNLPam. Directora del Instituto de Ciencias de la Educación para la investigación interdisciplinaria. Facultad de Ciencias Humanas. UNLPam. Editora Científica de la Revista del ICEII Praxis Educativa. Directora del Programa Formación docente: una práctica profesional reflexiva en los profesorados universitarios y del Proyecto El Campo de la Práctica: una arquitectura innovadora que recupera el valor formativo de la práctica en los profesorados universitarios.

3 Profesora en Geografía (UNLPam). Especialista en Estudios Sociales y Culturales (UNLPam). Docente de Residencia Docente del Profesorado en Geografía de la Facultad de Ciencias Humanas de la UNLPam. Responsable de la articulación e implementación del Campo de la Práctica del Departamento de Geografía. Co- Directora de los Proyectos de Investigación La enseñanza de la Geografia: aportes teóricos, currículum, prácticas, sujetos y Sociedad, economía y ambiente. Perspectivas teóricas, análisis de casos y estrategias didácticas, en el marco del Programa "Contextos territoriales contemporáneos: abordajes desde la Geografía". Miembro integrante de la "Red de Docentes Investigadores en la enseñanza de la Geografia" (ReDIEG). 
Docente. A la que se suma el Campo de la Práctica, "Prácticas destinadas al aprendizaje sistemático de las capacidades para la actuación docente en las aulas y en los distintos ámbitos en donde se desempeñe profesionalmente" (Res. 232-CS-2009).

Este Campo se planifica desde el primer año de la carrera, no se organiza en asignaturas, sino que se configura como un eje transversal que apunta a que cada materia o grupos de materias vincule conocimientos y competencias orientadas a la enseñanza de la Geografía, con el propósito de superar la distancia habitual que existe entre formación disciplinar y pedagógica.

La novedad de este formato curricular lo constituye la presencia de un Campo de las Prácticas que nace desde el primer año de la carrera. Se confirma que aparece por primera vez en el recorrido histórico-curricular a partir del análisis de los distintos planes de estudios del Profesorado de Geografía (1962, 1967, 1971, 1976, 1984, 1994, 1998, 2003 у 2009), poniendo en foco la relación establecida en ellos entre teoría y práctica educativa. Del análisis del lugar formal de la práctica en el recorrido histórico curricular del Profesorado de Geografía (UNLPam), podrían señalarse que ha predominado en todos los planes una concepción técnica. Se advierte la presencia de la práctica en el/los últimos años de la carrera, en asignaturas como Metodología Especial y Residencia con la idea de transferir al contexto del aula los saberes construidos en las asignaturas de los años anteriores. Se descansa sobre una teoría que anticipa y determina a la práctica. $\mathrm{La}$ autoridad es portada por quien posee esos saberes y definen los contenidos a enseñar, porque son valorados en la universidad. Se lleva adelante en el último año de la carrera, porque se han consolidado mayoritariamente los saberes teóricos necesarios para la enseñanza (Camilloni, 2009; Litwin, 1995). La formación docente es fortalecida como destreza, como un entrenamiento en métodos y técnicas, cuántas más habilidades se le oferten en la universidad, mejor será el desempeño docente que podrá ofrecer un saber externo, universal, ahistórico (Grundy, 1991). Una formación disciplinar sólida, conocer las características evolutivas de los estudiantes con quien se trabajará y una didáctica de la disciplina desarrollada entre la institución formadora y la escuela, constituyen una triangulación potente para dar clases en la escuela secundaria (Davini, 2009).

Este recorrido documenta que el nuevo plan reconoce el valor formativo de la práctica desde el inicio de la carrera en la búsqueda del aprendizaje sistemático de las capacidades para la actuación docente en las aulas. De este modo, el plan incorpora a aquellas actividades o procesos de la formación que los/as estudiantes universitarios/as realizan fuera de la universidad en contexto de profesiones reales desde el inicio de la carrera. 


\section{Los desafíos del Campo de la Práctica}

Los desafíos del Campo de la Práctica pasan por cómo proponer en el territorio concreto, focalizado en la institución escuela, un trabajo con los actores locales que signifique desde la enseñanza de la Geografía, la construcción de ciudadanía como proyecto que reconoce la multiplicidad de voces.

En este sentido, es fundamental fortalecer el vínculo entre docentes y estudiantes del Profesorado y la escuela como espacio de construcción permanente, de generación de confianza, de reciprocidad y de constitución de un entramado que reconoce el capital social y cultural de los territorios y los actores. Esto requiere la incorporación progresiva de las asignaturas en un lento proceso político pedagógico, que necesita dejar de mirar a las escuelas como espacios extraños y amenazantes. Asimismo implica construir horizontalidades con docentes de Geografía que buscan romper el aislamiento por medio de la utilización adecuada de los contenidos propuestos en términos de una perspectiva vertical y fragmentada. Todo esto supone un posicionamiento docente frente a la formación y a los saberes geográficos, que va más allá de lo instrumental, para constituirse en prácticas que necesitan de un complejo proceso pedagógico, ético, político y cultural.

¿Cómo involucrarnos en el trabajo colectivo que implica esta tarea?

En coincidencia con el documento propuesto por ANFHE (2016), las prácticas profesionales docentes son prácticas sociales e históricas que responden a intenciones y valores determinados por los actores que en ellas intervienen, en cada momento y circunstancia en que se desarrollan. Estas prácticas se fundamentan en concepciones y valoraciones que nutren la acción, en las que teoría y práctica son mutuamente constitutivas en una interacción permanente.

El mismo otorga especificidad a las asignaturas que lo conforman como un trayecto formativo que posibilita a los/ las estudiantes una aproximación a su campo de intervención profesional y al conjunto de las tareas que le corresponderá asumir. En tal sentido, compromete una doble intelección: la descripción, análisis e interpretación de la complejidad y multidimensionalidad que caracteriza las prácticas docentes y de la enseñanza y los contextos en las cuales se inscriben; es decir, la orientada a la comprensión de las condiciones objetivas de producción y las que remiten al sujeto de estas prácticas, a la implicación de su subjetividad e identidad al incluirse desde una historia y una trayectoria singular; cuestión que reclama procesos de objetivación mediados desde una posición de reflexividad crítica. 
Entre las actividades desarrolladas que hacen al ejercicio profesional docente: oobservación y análisis de la inserción institucional del profesor universitario, análisis de documentos curriculares, observación, registro y análisis de clases, análisis de materiales didácticos, libros de texto, documentos electrónicos y software específico, elaboración, puesta en práctica y análisis de propuestas de enseñanza y aprendizaje en diferentes contextos, análisis de producciones de los alumnos como insumo para la elaboración de propuestas didácticas, participación en procesos de evaluación de los aprendizajes, uso de las TIC como herramientas para la enseñanza y el aprendizaje de la disciplina, análisis de contenidos a enseñar en función del nivel en el que serán desarrollados y del proyecto educativo institucional, estuvieron presentes como actividades propias del campo de la formación docente, a partir de esta propuesta que nos convoca a todos se les otorga otro sentido que resignifica las prácticas. Además, los aprendizajes de los estudiantes se amplían y enriquecen cuando nos miran como docentes en instancias de diseño de propuestas, en las cuales ponemos en juego ideologías, perspectivas, concepciones, las cuales eran observadas en cada espacio de enseñanza como actividades fragmentadas. Y aquí una reflexión, los estudiantes tienen necesidad de encontrarnos como docentes en la construcción de lo común que es la formación docente.

\section{Un mapa de situación}

En este sentido, el camino recorrido nos permite pensar que la prescripción que implicó la incorporación del Campo de la Práctica en el Plan de Estudios 2009 se aproxima a su institucionalización a través de acuerdos entre docentes de Geografía de las Universidades Públicas. Las experiencias realizadas en nuestra facultad han trascendido el ámbito de las aulas y las escuelas, porque han sido compartidos a través de las voces de estudiantes y docentes en eventos académicos. El reconocimiento de la propuesta alienta a todos a continuar este proceso de articulación en la búsqueda de la integralidad.

Esto requiere articulaciones múltiples y diversas para romper con las representaciones del adentro y afuera en el ámbito educativo, y favorecer el trabajo con diferentes organizaciones sociales. Asimismo proporcionar, a los docentes en formación, las herramientas conceptuales y metodológicas que les permitan el acceso a los más diversos ámbitos de la cultura, y desde allí elaborar diseños referenciales que propicien y vertebren el ejercicio de trabajo docente responsable y comprometido. 
Las propuestas realizadas desde los diferentes campos de formación del Profesorado en Geografía, hicieron hincapié en la formación específica y docente, en las que explicitaron una amplia participación con variadas prácticas, organizadas en tres formatos:

\section{- Prácticas al interior de la asignatura}

Estas propuestas constituyen una modalidad organizativa centrada en vincular los saberes disciplinares. Se desarrollan en distintos momentos de la cursada, y en asignaturas de diferentes años del plan. Crean fuerte pertenencia de grupo y fuerte identidad respecto a la materia. Ponen en foco saberes que son estructurantes en el contexto de la escuela. Otras establecen vínculos entre los contenidos de las asignaturas y elementos escolares (libros de texto de enseñanza secundaria, materiales curriculares, recortes periodísticos, estadísticas escolares), con actores de la escuela (entrevista a directivos, docentes, referentes gremiales).

\section{- Prácticas de articulación entre asignaturas}

Las propuestas analizadas desde esta perspectiva articulan dos o más asignaturas del plan de estudio, ambas de la formación disciplinar, general o pedagógica. Las zonas de enlace pueden privilegiar temáticas compartidas, autores seleccionados, territorios analizados, escalas, problemáticas, actores buscando equilibrar la presencia de las asignaturas vinculadas. La intersección colabora en mejorar las relaciones al interior del propio plan de estudio. Constituye un nivel de integración mayor al anterior, presupone una vinculación que forma una zona intersticial compartida. El enlace es una idea relacionante, una estructura, o un concepto superior a un contenido, el cual se enfoca sobre principios generales lo que implica un mayor nivel de abstracción.

Los límites de las materias parecen más borrosos, con fronteras más débiles (Bernstein, 1974) lo que favorece una mayor integración multi o pluridisciplinar. Salidas de campo, viajes, análisis de casos, ateneos, constituyen las modalidades más frecuentes de intervención. En general, se han llevado adelante avanzadas las cursadas, en tanto se han desarrollado las temáticas y luego han realizado la propuesta compartida. Requiere un trabajo docente de alto nivel de relación en las tareas educativas y establece mejores vinculaciones con los contextos sociales y culturales. En esta modalidad como en la anterior, los/as estudiantes valoran que las propuestas han fortalecido la comprensión conceptual de la asignatura. 


\section{- Prácticas vinculadas a la escuela}

Las propuestas llevadas adelante en relación a la escuela tienden a resignificar los conocimientos de los otros espacios curriculares, ya que prepara a los/as estudiantes para una participación e incorporación progresiva en distintos contextos socio-educativos.

Distintas propuestas se planifican en este sentido desde salidas y visitas diarias hasta períodos de inmersión de un cuatrimestre en campo. Entre las primeras, el contacto a realidad, vínculo con actores en entrevistas, observación, diálogos, juegos. Hay actividades en las que planifican clases, análisis de casos, salidas con estudiantes secundarios, problemáticas de interés. Estas propuestas tienen doble relación, algunas han sido solicitadas por las/os graduados y otras las planean las cátedras universitarias. Son las mejores evaluadas, en tanto fortalecen la contextualización, la enseñanza situada, la vinculación con la profesión, los contenidos universitarios constituyen conocimientos en acción, dado que se trata de situarse en el contexto y analizar cómo intervenir en ellas. En este sentido, se propone que la cultura escolar no se comprenda como una aplicación de la cultura científica, dado que tienen muy distinta finalidad; y favorece aprender a reflexionar sobre los procesos de construcción se subjetividad, de identidad profesional, de relación entre sociedad y escolarización, entre teoría y práctica, entre formación disciplinar y formación pedagógica.

En este camino recorrido los dispositivos de integración más potentes implementados en estas experiencias son:

- Salidas de campo: en este dispositivo, los/as profesores/as del Profesorado de Geografía generan una posibilidad de articular saberes, asignaturas, vínculos con la realidad y con el sentido de la formación, todos aspectos que fortalecen la comprensión y la transferencia. Este trabajo compartido tiende a la reducción de la balcanización de la enseñanza universitaria (Hargreaves, 1999). Se potencia el trabajo con la estructura semántica y sintáctica del saber (Schwab, 1963; Litwin, 1995; 2013).

- Experiencias de intervención áulicas: este dispositivo se implementa por un número importante de asignaturas $\mathrm{y} / \mathrm{o}$ grupo de asignaturas que diseñan estrategias de enseñanza en clase secundarias. Pueden ser en el desarrollo de temáticas, análisis de casos, propuestas alternativas, clases especiales, entrevistas en aula. De este modo se generan prácticas concretas, breves en tiempo escolar delimitado (una o varias clases), se refleja la implicación personal y académica en la elaboración del saber a enseñar. Estas acciones buscan confirmar un pensamiento práctico 
que involucra el conocimiento que está en la acción, para luego reflexionar sobre la acción.

- Ayudantías: son un dispositivo de formación, investigación y reflexión diseñado para que los/as estudiantes de profesorado consoliden aprendizajes sistemáticos de las capacidades de la actuación docente en contexto situados y complejos. Se llevan adelante en el aula escolar durante el período que dure la cursada universitaria o parte de ella gestionan las tareas que hace un ayudante, de manera similar a las que experimentan en la Facultad: acompañan al profesor en tareas de diseño, organización, selección, búsqueda de materiales, de casos, de ejemplos.

En la particularidad de cada experiencia hay quienes tuvieron la posibilidad de acompañar grupos, hacer explicaciones a algún alumno con dificultad hasta preparar y llevar adelante un práctico, un teórico planeado con el profesor a cargo del espacio curricular, diseñando evaluaciones, sugiriendo criterios de evaluación, compartiendo la corrección con los/ as docentes de aula, siempre con la prioridad de ayudar a profesores/as y alumnos/as que presenten mayor dificultad (Di Franco, 2016).

\section{Significados construidos en relación del Campo de la Práctica y de la práctica}

Los nuevos planes diseñados han puesto el foco en la práctica educativa, que se constituye en formación relevante si podemos pensar la práctica como constitutiva de la formación de profesores. Por este motivo, se ha buscado esclarecer de qué se habla en términos de prácticas y del campo de la práctica.

El proceso analítico de investigación en la acción recupera los significados en construcción respecto al Campo de la Práctica. En este sentido, se llevan adelante reuniones en distintos momentos de las cursadas para diseñar y compartir propuestas implementadas, instancias de evaluación, entrevistas a docentes, encuestas a estudiantes. De este modo, se pretende entender al plan no como una prescripción cerrada, sino como un dispositivo pedagógico orientado por hipótesis provisionales que muevan a la acción.

De los registros de las voces quedan documentados que en el campo de la práctica se vinculan saberes teóricos con el contexto de intervención profesional (P.I). En varios casos el campo de la práctica es visto como 
herramienta para conflictuar conceptos previos construidos por los/as estudiantes, que puede hacerse mejor en el contexto real. Otro de los significados atribuidos, es que favorece mejores articulaciones entre asignaturas del plan, tanto disciplinares como de la formación docente (PDE; PD; PS). Se suma quien lo entiende como un vínculo con el ámbito de trabajo docente, que anticipa qué sucede en las modalidades de trabajo e identidad profesional en campo (PHA). En la mayoría de los casos ya se incorpora como una instancia de proceso de enseñanza y aprendizaje de la asignatura, que permite a los estudiantes articular los conceptos y procesos que abordan en las clases teóricas y prácticas con el contexto situado, ver cómo aprenden los diferentes grupos en situaciones diferentes, qué estrategias de intervención pueden ser más oportunas en cada contexto.

Respecto al significado de práctica, teoría y práctica constituyen una relación fundamental en la formación de profesoras/es. Varios autores abordan estos argumentos desde distintas racionalidades técnica, práctica y crítica (Grundy, 1991); perspectiva de la oposición entre teoría y práctica; dependencia de la práctica de la teoría; práctica independiente de la teoría (Carr, 1996).

En las voces docentes: práctica es aplicación (PA); es la puesta en marcha de la experiencia inicial en la transposición (PB); práctica es empiria mediada por teoría (PI); práctica exige tomar decisiones, elegir fundadamente (PHA); práctica es construcción colectiva, compartida con otras cátedras y personas, como proyecto institucional poniendo en situación y en diferentes contextos al saber geográfico (PU -PAL); práctica es praxis ( $\mathrm{PD}$; $\mathrm{PDE}$ ); práctica es reciprocidad entre teoría y práctica (PS; PGA-E); práctica es reflexión de la acción para construir teoría (PRD).

Estas diferencias en la conceptualización es imprescindible explicitarlas para reducir los niveles de contradicción. Los aportes docentes en relación al sentido de las prácticas y sus aprendizajes permiten teorizar acerca de la formación necesaria para que el saber pueda ser enseñado y reconstruido.

\section{Para seguir pensando}

El propósito fue habilitar propuestas en el Campo de la Práctica para fortalecer los acuerdos sobre las categorías conceptuales que estructuran los contenidos de las asignaturas y posibilitan la conformación de tramas conceptuales, teniendo en cuenta la complejidad, la articulación y el tratamiento de las múltiples dimensiones de análisis, que orientan hacia la construcción de un enfoque territorial crítico. 
Estas prácticas han logrado un lugar legitimado en relación a su incidencia en los procesos de formación, que se materializa en el tramo final. De este modo, se constituye en una instancia favorable para socializar experiencias y desarrollos conceptuales para profundizar en los avances.

En coincidencia con Henry Giroux (2014), los docentes debemos ubicar las nociones de crítica y conflicto en el centro de los modelos pedagógicos. Dentro de esta perspectiva existen mayores posibilidades para desarrollar una comprensión del papel que desempeña el poder de definir y distribuir el conocimiento y las relaciones sociales que median entre la escuela y las experiencias en las aulas. La crítica debe llegar a ser herramienta pedagógica vital, no sólo porque irrumpe en las mistificaciones y distorsiones que trabajan desde las rutinas de las prácticas, sino también porque modela una forma de resistencia y de pedagogía de oposición.

Para ello, es necesario analizar las relaciones de poder, que según Foucault (1976) no es un fenómeno estático, por ese motivo analizarlas en la escuela, debería permitirnos desarrollar prácticas pedagógicas alternativas.

Michael Apple (2000) en construir escuelas democráticas señala que esto no significa que otras fuentes de conocimiento profesional sean inválidas o resulten inútiles; sencillamente significa que no son las únicas fuentes de ideas que merecen la pena. Cuando relacionamos el derecho democrático de los docentes a ejercer un control significativo sobre su propio trabajo con la obligación por parte de los profesores y otros adultos de extender la forma de vida democrática a los jóvenes, vemos la posibilidad real de que los valores democráticos puedan convertirse en una fuente de coherencia para la vida en nuestras escuelas. Sin embargo, para convertir la posibilidad en realidad, tendremos que tropezarnos con algunas preguntas difíciles.

En este sentido, "nos aguarda la inmensa tarea de repensar continuamente en qué consiste el oficio del profesor de geografía en el actual contexto sociopolítico, atendiendo a la complejidad del trabajo del aula, a los significados de las culturas y las prácticas juveniles, y a las siempre cambiantes circunstancias históricas, materiales y simbólicas, en las que desenvolvemos nuestras vidas y nuestros trabajos" (Fernández Caso y Gurevich, 2014, p. 29).

Como desafío es preciso ampliar y profundizar prácticas de enseñanza de geografía mediante estrategias alternativas enmarcadas en la propuesta de formación integral, que se retroalimente a partir de las experiencias de la práctica desde la perspectiva del campo de formación profesional. Esto significa fortalecer las dimensiones social y crítica con el compromiso de docentes y estudiantes, para superar la fragmentación teoría-práctica, y promover prácticas desde un proceso formativo con sentido ético y político. 


\section{Bibliografía}

Apple, M y J. BeAne (comps.) (2000) Escuelas democráticas. Madrid: Morata.

Bernstein, B. (1974). Clases, códigos y control, Vol. 1 London: Routledge and Kegan Paul.

CARr, W y Kemmis, S. (1988) Teoría crítica de la enseñanza. La investigación acción en la formación del profesorado. Barcelona, Martínez Roca.

Camilloni, A. R. W. (2001) "Modalidades y proyectos de cambio curricular". Aportes para el cambio curricular en Argentina 2001. Buenos Aires: Universidad de Buenos Aires. Facultad de Medicina. OPS/OMS.

CAmilloni, A. (2007). Justificación de la Didáctica. Por qué y Para qué la Didáctica. En Camilloni, A.; Cols, E.; Basabe, L. y otra 2007. El saber didáctico. Buenos Aires: Paidós.

Davini, M. C. (2009). Métodos de enseñanza. Didáctica general para maestros y profesores. Buenos Aires: Ed. Santillana.

Fernández Caso. M. V y Gurevich. R. (2014). Didáctica de la geografia. Prácticas escolares y formación de profesores. Buenos Aires: Editorial Biblos.

Foucault, M. (1976). Vigilar y castigar. Nacimiento de la prisión. México: Siglo XXI.
Giroux, H. (2014) .Teoría y resistencia en educación. México: Siglo veintiuno editores. Centro de Estudios sobre la Universidad, UNAM. $8^{\circ}$ reimpresión.

Grundy, S. (1991). Producto o Praxis del curriculum. Madrid: Morata.

Hargreaves, A. (1999). Profesorado, cultura y posmodernidad. Cambian los tiempos, cambia el profesorado. Madrid: Morata.

Kemmis, S. (1988). El currículum: más allá de la teoría de la reproducción. Madrid: Morata.

Litwin, E. (1995). Prácticas y teoría en al aula universitaria en Revista Praxis Educativa Facultad de Ciencias Humanas, UNLPam, Año I, $\mathrm{N}^{\mathrm{o}} 1$.

PÉREZ Gómez, A. (2004). La cultura escolar en la sociedad neoliberal. Madrid: Morata.

Sснӧn, D. (1988). El profesional reflexivo. Cómo piensan los profesionales cuando actúan. Barcelona: Paidós.

Souto, M. y OTROS. (1999). Grupos y dispositivos de formación. Buenos Aires: Novedades Educativas.

Stenhouse, L. (1987). Investigación y desarrollo del currículum. Madrid: Morata. 